\title{
Integrating a Health Coach into Primary Care: Reflections From the Penn State Ambulatory Research Network
}

\author{
Alan M. Adelman, MD, MS, Marie Graybill, RN \\ Department of Family \& Community Medicine, Penn State Ambulatory Research Network, Hershey, Pa
} Ann Fam Med 2005;3(Suppl 2):S33-S35. DOI: 10.1370/afm.317.

Conflicts of interest: none reported

CORRESPONDING AUTHOR

Alan M. Adelman, MD, MS, Department of Family \& Community Medicine, Penn State Ambulatory Research Network, 500 University Dr, H154, Hershey, PA 17033, aadelman@psu.edu

\section{PURPOSE}

I n our Promoting Healthy Behaviors in Primary Care project, we proposed to (1) develop a health coach model of behavior change, integrated into primary care practices to improve diet and increase physical activity among obese adults, and (2) evaluate the effectiveness of a motivational program delivered by the health coach to assist patients in improving their unhealthy behaviors.

\section{METHODS}

In 4 family practice sites, the nursing staff and clinicians were asked to identify patients who were obese (body mass index >30) and invite them to participate in a program that would offer a personalized approach for addressing poor diet, physical inactivity, or both. Potential study patients were referred to an on-site health coach who (1) described the project and obtained informed consent ${ }_{i}(2)$ collected baseline demographic and lifestyle data ${ }_{i}$ (3) gave My Healtby Habits Journal ${ }^{1}$ to each participant ${ }_{i}(4)$ provided regular follow-up to enrollees; (5) offered additional educational materials on motivation, diet, and physical activity; and (6) when appropriate, linked patients to community resources. The lay health coach provided follow-up support via face-toface visits, e-mail, telephone contacts, or some combination thereof on average every 2 to 4 weeks for up to 6 months. At the close of the study, participants were asked to complete a follow-up questionnaire and exit interview.

The intervention focused on patients working through My Healthy Habits Journal with the health coach to develop an individualized action plan to address poor diet, physical inactivity, or both. This motivational enhancement approach proposed by Miller and Rollnick and Botelho ${ }^{3,4}$ is largely based on motivational interviewing (MI). MI is "a directive, client-centered counseling style for increasing intrinsic motivation by helping clients explore and resolve ambivalence. ${ }^{15}$ It is based on 5 guiding principles: expressing empathy, developing an awareness of the discrepancy between the patient's present state and his or her goal, "rolling" with the patient's resistance to change, avoiding arguments, and supporting self-efficacy.

The initial 4-week training program for the health coach consisted of review of videotapes, ${ }^{2}$ reading assignments, ${ }^{3,4,6}$ a self-change project, and practice sessions with 2 patient participants. The trainer reviewed audiotapes of the practice sessions and provided feedback via telephone and e-mail. Once participant recruitment began, ongoing training through review of audiotaped interactions was by telephone, e-mail contact, or both every 1 to 2 weeks between the health coach and trainer. In the latter stages of the project, the transcribed audiotapes were rated using the Motivational Interviewing Treatment Integrity (MITI) scale. ${ }^{7}$ The MITI scale counts therapist behaviors that are important to MI, such as validating the patient's feelings, asking open-ended questions, and listening reflectively. It also counts behaviors that do not adhere to MI, such as directing or persuading the patient, and giving unsolicited advice.

\section{LESSONS LEARNED}

Use of this model of an on-site health coach trained in motivational techniques led to 44 (48\%) of the 92 patients initiating a behavior change in eating habits, physical activity, or both. Most patients preferred face-to-face meetings to telephone or e-mail contacts. Review of the audiotapes, exit interviews, and e-mail communications between the health coach and patients revealed a positive, supportive exchange. Staff at each practice site unanimously expressed the energizing impact of the health coach on patients and staff. Through involvement in the project, many office staff became enthusiastically committed to their own personal healthy lifestyle behavior change.

Our study suggests that health coaches require careful caseload management and structured guidelines to address setting boundaries. Boundary issues arose 
because of the frequent occurrence of psychosocial issues including depressive symptoms in this population of obese adults. Many patients readily divulged their deeply personal struggle surrounding their eating habits. In turn, the health coach often struggled with how best to address these consuming psychosocial issues. In the future, further attention should be given to recognition of the many psychosocial complexities that compete with facilitating behavior change. In addition, firm boundaries must be set as to what the health coach can address and how much time is spent with patients who are not actively modifying their unhealthy behaviors.

Central to this project was the effective implementation of motivational techniques by the lay health coach. Although we did not directly examine what characteristics are needed to be an effective health coach, the individual must possess strong negotiating and listening skills and the capacity to project a nonjudgmental, supportive presence. The rate of adoption and implementation of the motivational enhancement techniques by the health coach depended on the particular technique and its difficulty. Learning to ask open-ended questions was relatively easy, whereas decreasing the number of closed-ended questions was more difficult. Similarly, validating the patient's feelings and providing emotional support were easier to master, whereas decreasing the use of persuasion and direct advice took longer to adopt. The rate of uptake of the skills necessary to be an effective health coach probably varies depending on the coach's baseline skills and personal style. For the more difficult skills, 9 to 12 months was required to master their use. Unfortunately, the literature on MI does not provide guidance on length of time required to master all the skills. In teaching MI to mental health or addiction counselors, the use of standardized patients has been found to be beneficial. ${ }^{8}$ The use of standardized patients for structured practice may decrease the time required for learning these techniques. This project suggests that lay health coaches require intensive, interactive training and timely supportive feedback.

Implementation is 1 of the 5 key elements proposed by Glasgow et $\mathrm{al}^{9}$ in their RE-AIM framework for translational research. Also called treatment integrity or fidelity, implementation refers to the quality and consistency of the intervention in a real-world setting. If an intervention fails, the question arises whether the failure was due to an ineffective intervention or to inappropriate delivery of the intervention. Glasgow et $\mathrm{al}^{10}$ reported that behavioral interventions may be more difficult to evaluate because of the "inherent interactivity" that characterizes the patient and health coach relationship.

Two other issues surrounding the training of the health coach had an important impact on implementation. First, audiotaping health coach-patient interactions was an essential part of this project. Traditionally, MI training occurs in workshop format. After workshop training, self-reported knowledge increases, but newly acquired skills may not be incorporated into practice when measured at a 2- to 4-month follow-up. ${ }^{8,11}$ Continued evaluation of the intervention through the use of video or audiotape is a valuable way to monitor the training process and ensure uniformity of the intervention.

Second, during the last 3 months of the project, the adoption of a standardized measure (MITI) to evaluate the health coach's performance was a valuable training aid. Feedback was structured around specific health coach behaviors that needed to be increased or decreased. The recently described behavior change counseling index $(\mathrm{BECCI})^{12}$ appears to be more aligned with brief interventions and could replace the MITI as the instrument of choice to measure the delivery of motivational enhancement techniques.

\section{CONCLUSIONS}

An important lesson learned from this pilot project was that brief motivational enhancement techniques provided by a lay health coach could be successfully implemented in the primary care setting. To ensure that health coaches deliver the intended behavioral interventions, training must be carefully planned and monitored. Further research is needed to determine the optimal teaching strategies that will enhance the integration of motivational enhancement techniques into practice.

To read or post commentaries in response to this article, see it online at http://www.annfammed.org/cgi/content/full/3/Suppl_2/S33.

Key words: Practice-based research network; health behavior; health promotion; obesity; motivation

Submitted December 20, 2004; submitted, revised, March 13, 2005; accepted March 23, 2005.

Funding support: This project was supported by Prescription for Health, a national program of The Robert Wood Johnson Foundation with support from the Agency for Healthcare Research and Quality.

Acknowledgments: Drs Rick Botelho, John George, and Deborah Van Horn are appreciated for their helpful comments on earlier versions of the manuscript. Drs Botelho and Van Horn also served as trainers of the health coach. The clinicians and staff of the University Physician Group practices of Elizabethtown, Fishburn, Front Street, and Palmyra are appreciated for their participation in this project. Judy Doherty, MA, is acknowledged for her efforts and dedication as the health coach. Connie Zimmerman is acknowledged for her tireless effort in transcribing the audiotapes.

\section{References}

1. Botelho RJ. My Healthy Habits Journal: Stepping Stones to Lasting Change. Rochester, NY: MHH Publications; 2003:64.

2. Miller WR, Rollnick S. Motivational Interviewing: Preparing People for Change. 2nd ed. New York, NY: Guilford Press; 2002. 
3. Botelho R. Motivate Healthy Habits: Change Yourself Before Helping Others. Rochester, NY: MHH Publications; 2002.

4. Botelho R. Beyond Advice: 2. Developing Motivational Skills. Rochester, NY: MHH Publications; 2002.

5. Miller W/R, Rollnick S, Moyers TB. Motivational Interviewing. Professional Training Videotape Series. Albuquerque, NM: Center on Alcoholism, Substance Abuse and Addictions, University of New Mexico; 1998.

6. Botelho R. Beyond Advice: 1. Becoming a Motivational Practitioner. Rochester, NY: MHH Publications; 2002.

7. Moyers TB, Martin T, Manuel JK, Miller WR. The Motivational Interviewing Treatment Integrity (MITI) Scale. Version 2.0. Albuquerque, NM: Center on Alcoholism, Substance Abuse and Addictions, University of New Mexico; 2003.

8. Baer JS, Rosengren DB, Dunn CW, Wells EA, Ogle RL, Hartzler B. An evaluation of workshop training in motivational interviewing for addiction and mental health clinicians. Drug Alcohol Depend. 2004;73:99-106.
9. Glasgow RE, Vogt TM, Boles SM. Evaluating the public health impact of health promotion interventions: the RE-AIM framework. Am J Public Health. 1999;89:1322-1327.

10. Glasgow RE, Lichtenstein E, Marcus AC. Why don't we see more translation of health promotion research to practice? Rethinking the efficacyto-effectiveness transition. Am J Public Health. 2003;93:1261-1267.

11. Miller WR, Mount KA. A small study of training in motivational interviewing: does one workshop change clinician and client behavior? Behav Cogn Psychother. 2001;29:457-471.

12. Lane C, Huws-Thomas M, Hood K, Rollnick S, Edwards K, Robling $M$. Measuring adaptations of motivational interviewing: the development and validation of the behavior change counseling index (BECCI). Patient Educ Couns. 2005;56:166-173.

\title{
Wellness for a Lifetime: Improving Lifestyle Behaviors of Low-Income, Ethnically Diverse Populations
}

\author{
Laura Anderko, PbD, RN, Claudia Bartz, PbD, RN, FAAN, Sally Lundeen, PbD, RN, FAAN \\ University of Wisconsin-Milwaukee College of Nursing, Milwaukee, Wis \\ Ann Fam Med 2005;3(Suppl 2):S35-S36. DOI: 10.1370/afm.318. \\ Conflicts of interest: none reported
}

CORRESPONDING AUTHOR

Laura Anderko, PhD, RN, University of Wisconsin-Milwaukee College of Nursing, PO Box 413, Milwaukee, WI 53202-0413, landerko@uwm.edu

\section{PURPOSE}

$\mathrm{E}$ ight community nursing centers (CNCs) belonging to the Midwest Nursing Centers Consortium, a practice-based research network, implemented a community-based, multidimensional strategy to promote behavior changes among high-risk, ethnically diverse, low-income adults. The purposes of this study were (1) to promote increased physical activity levels and improved nutrition among CNC clients, and (2) to assess clinician evaluation and referral behaviors regarding clients' physical activity and nutrition. This article describes key lessons learned from this study.

\section{METHODS}

The study used a time-series design across the 8 CNCs. The study was grounded in a community-based, public health framework, thus encouraging community outreach for participant recruitment. Clients were recruited for a 16-week course titled Wellness for a Lifetime. The course materials were based on a 10 -week pilot project recently completed by one of the CNCs. These materi- als, developed by a multidisciplinary team using health behavior theory ${ }^{1}$ and culturally appropriate content, were provided to each $\mathrm{CNC}$ and outlined didactic course content, as well as themes used for discussion during the support group sessions. Didactic topics included food categories and nutritional content; principles of stretching and exercising; relationship of disease, such as hypertension, to diet and activity; and methods of stress reduction. Participants practiced safe exercises and used workout plans and walking routes. Support sessions included discussion of challenges and successes related to behavior changes, as well as field trips to fast-food restaurants and grocery stores to learn how to determine the nutritional content of a variety of food items.

Data were collected and analyzed from (1) chart audits (preintervention and postintervention) to assess change by advanced practice nurses (APNs) in documentation of assessments and in referrals for clients' nutrition and physical activity; (2) end-of-program telephone interviews of APNs at the 8 sites to identify barriers to recruitment and retention, and strengths of the program from the clinicians' point of view; (3) weekly and end-ofcourse participant satisfaction surveys that included self- 